\title{
Plane-Projection Multi-Photon Microscopy for High-Frame-Rate Live Tissue Imaging
}

\author{
Jiun-Yann Yu' ${ }^{1}$, Chun-Hung Kuo², Ruben Zadoyan², and Chin-Lin Guo ${ }^{1}$ \\ ${ }^{1}$ Bioengineering, California Institute of Technology, 1200 E. California Blvd., Pasadena, California 91125, USA. \\ Tel: 626-395-5992. \\ ${ }^{2}$ Technology and Applications Center, Newport Corporation. \\ jyyu@caltech.edu
}

\begin{abstract}
We present a wide-field multi-photon microscopy that provides optical sectioning at high frame rate under biocompatible laser dosage. Axial resolution comparable to confocal microscopy and 5-frame-per-second live tissue imaging are demonstrated.
\end{abstract}

(C) 2011 Optical Society of America

OCIS codes: (180.4315) Nonlinear microscopy; (140.7090) Ultrafast lasers; (170.2520) Fluorescence microscopy; (190.4180) multi-photon processes.

\section{Introduction}

The two main advantages of wide-field fluorescence microscopy over laser scanning microscopy are the much lower excitation intensity at similar acquisition rate and the simplicity of the optical setup. The strong excitation intensity of laser scanning microscopy is due to the extremely short dwell time per pixel in scanning microscopy, and therefore significant photo-toxicity or thermal mechanical damage is inevitable if a fast time-lapse microscopy is required [1-3].

Nevertheless, conventional epifluorescence microscopy provides poor optical sectioning due to strong out-of-focus excitations. Recently, a wide-field multi-photon microscopy possessing optical sectioning, referred to as temporal focusing, has been reported [4]. In this technique, ultrafast laser pulses were first chromatically dispersed and regained their coherence at the image plane, thereby creating the optical sectioning [4]. There are, however, two main issues in this technique. First, each blazed diffraction grating is fabricated for a specific wavelength. Exciting multiple fluorophores, as required for most biomedical studies, would need multiple gratings and hence increase the complexity of the system. Second, the signal level is extremely weak due to the inevitable reduction of multi-photon excitation efficiency in the setup. This leads to a significant reduction of the image acquisition rate. For example, the reported frame rate is 0.033 frames per second (fps) at cells stained with DAPI (a fluorescent dye for chromosome staining) [4], which is usually brighter than fluorescent protein expressed in live tissues.

Here, we present an approach to resolve the restrictions of single excitation wavelength and low acquisition rate in temporal focusing (Fig. 1 (a)). We overcome the limitation of single excitation wavelength by using an optical diffuser as the scatterer. Oron et al. have theoretically suggested a poor temporal focusing effect created by an optical diffuser, which they treated as a flat plane of points generating incoherent ultrafast pulses simultaneously [4]. However, taking into account the random time delay induced by the surface roughness of the diffuser, we found that optical diffuser can generate a reasonable temporal focusing by confining the multi-photon excitation efficiency within a few Rayleigh lengths. Meanwhile, assuming the $n$-photon excitation $\propto P_{p}^{n}$ ( $P_{p}$ : peak power of the laser pulses), we found that the fluorescence signal detected per unit time is proportional to $f^{1-n}$ ( $f$ : repetition rate) at a constant average power and pulse width. For example, reducing the repetition rate from $100 \mathrm{MHz}$ to $1 \mathrm{kHz}$ can increase the signal $10^{5}$ fold in two-photon excitation $(n=2)$. This enables high acquisition rate under biocompatible laser dosage. In this report, we experimentally verified these two predictions by measuring the axial resolution of the system and by obtaining optical sections of live epithelial tissue expressing cyan fluorescent protein at $5 \mathrm{fps}$.

\section{Results}

The axial resolution is determined by the full width of half maximum (FWHM) of the axial point spread function (PSF) obtained by imaging isolated fluorescent microspheres, and was found comparable to that of conventional confocal microscopy with the same objective (Fig. 1 (b), (c)). To examine if the setup can detect the signal from fluorescent proteins expressed in live tissues under biocompatible laser dosage, we performed optical sections of a hemispherical 
(a)

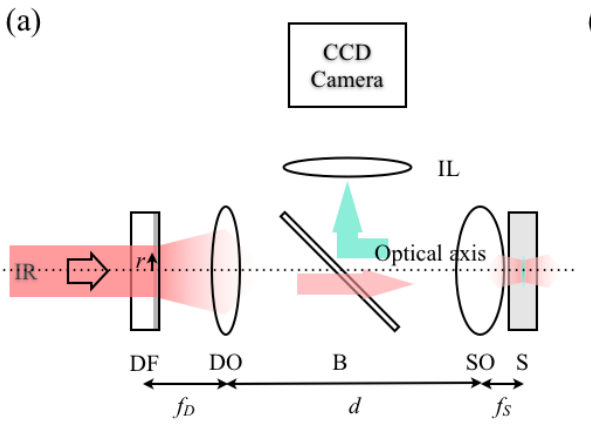

(b)

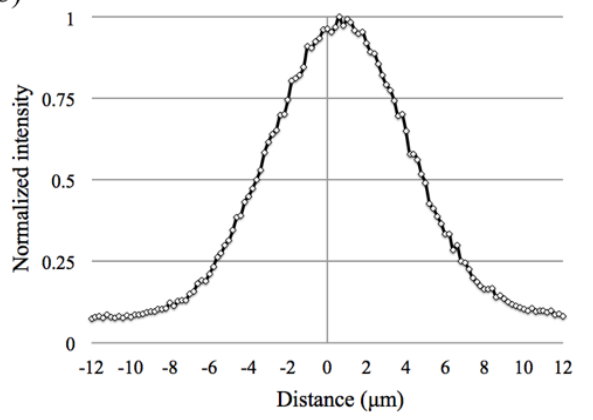

(c)

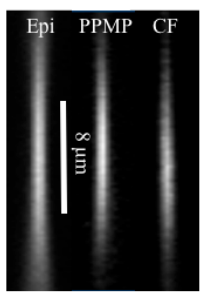

Fig. 1. (a) The setup of a plane-projection multi-photon microscope (PPMP). IR: $1 \mathrm{kHz}$ repetition rate ultrafast laser beam. DF: optical diffuser. DO: diffuser objective of focal length $f_{D}$. B: beamsplitter. SO: sample objective of focal length $f_{S}$. S: sample; IL: imaging lens. (b) Axial PSF by PPMP. 120 sectioning planes with a step size of $0.2 \mu \mathrm{m}$ were taken. Note that the FWHM here is $8.5 \mu \mathrm{m}$, while the theoretical axial resolution of a confocal microscope with the same SO is 7.2 $\mu \mathrm{m}$, and the Rayleigh length is $3 \mu \mathrm{m}$. Maximal excitation/emission of the microspheres: $430 / 465$ $\mathrm{nm}$. Diameter of the microspheres: $1 \mu \mathrm{m}$. SO: magnification $=10 \mathrm{X}, N A=0.3$. DO: magnification $=4 \mathrm{X}, N A=0.1$. Laser central wavelength, $790 \mathrm{~nm}$. (c) Lateral view of sectioning images from conventional epifluorescence microscopy (Epi), PPMP and conventional confocal microscopy (CF).

structure formed by live human mammary gland MCF-10A cells (Fig. 2). The frame rate was set 5 fps, comparable to that used in a conventional epifluorescence microscopy (100-500 ms) at the same sample. Our results suggest that the proposed setup possesses the capabilities of high-frame-rate acquisition and optical sectioning at the same time.

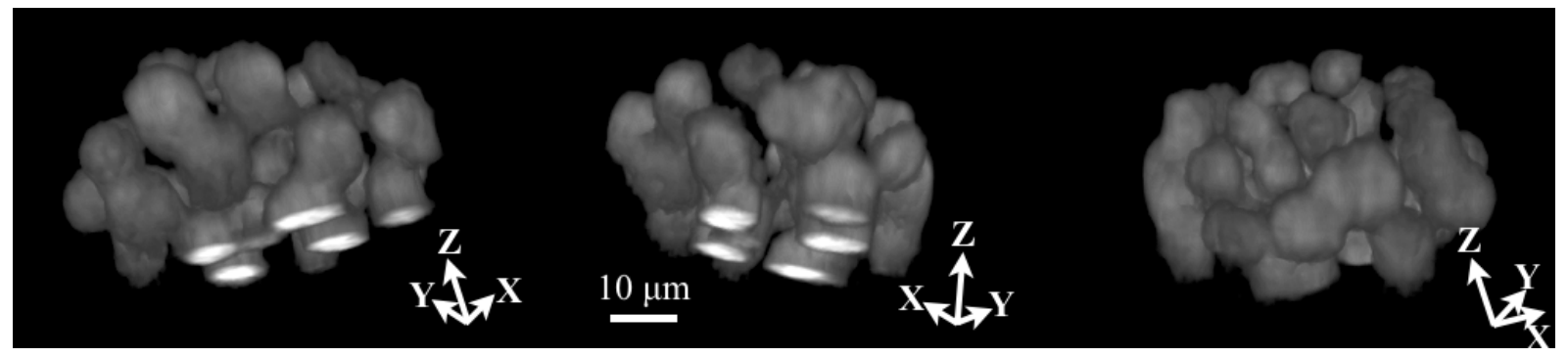

Fig. 2. Optical sections of live MCF-10A cells in a hemispherical structure. Fluorescence signals were from cell nuclei expressing cyan fluorescent protein-conjugated histone (H2B-cerulean) which binds to chromosomes. Exposure time of each frame: 0.2 seconds. Sample objective: $60 \mathrm{X}, N A=1.1$, $n=1.33$. Step size: $1 \mu \mathrm{m}$. Laser average power: $<10 \mathrm{~mW}$.

\section{References}

1. J. B. Pawley, Handbook of biological confocal microscopy (Springer, New York, NY, 2006), 3rd ed.

2. S. Potter, "Vital imaging: Two photons are better than one," Current Biology 6, 1595-1598 (1996).

3. B. Masters, P. So, C. Buehler, N. Barry, J. Sutin, W. Mantulin, and E. Gratton, "Mitigating thermal mechanical damage potential during two-photon dermal imaging," Journal of Biomedical Optics 9, 1265-1270 (2004).

4. D. Oron, E. Tal, and Y. Silberberg, “Scanningless depth-resolved microscopy,” Opt Express 13, 1468-76 (2005). 